
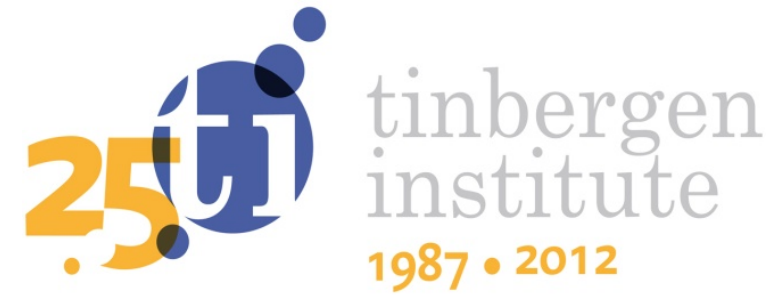

\title{
Associated Consistency Characterization of Two Linear Values for TU Games by Matrix Approach
}

\author{
Genjiu X $\mathbf{u}^{\top}$ \\ René van den Brink² \\ Gerard van der Laan² \\ Hao Sun ${ }^{1}$
}

${ }^{\prime}$ Northwestern Polytechnical University, Xi'an, P.R. China;

2 Faculty of Economics and Business Administration, VU University Amsterdam, and Tinbergen Institute. 
Tinbergen Institute is the graduate school and research institute in economics of Erasmus University Rotterdam, the University of Amsterdam and VU University Amsterdam.

More TI discussion papers can be downloaded at http://www.tinbergen.nl

Tinbergen Institute has two locations:

Tinbergen Institute Amsterdam

Gustav Mahlerplein 117

1082 MS Amsterdam

The Netherlands

Tel.: +31(0)205251600

Tinbergen Institute Rotterdam

Burg. Oudlaan 50

3062 PA Rotterdam

The Netherlands

Tel.: +31(0)10 4088900

Fax: $+31(0) 104089031$

Duisenberg school of finance is a collaboration of the Dutch financial sector and universities, with the ambition to support innovative research and offer top quality academic education in core areas of finance.

DSF research papers can be downloaded at: http://www.dsf.nl/

Duisenberg school of finance

Gustav Mahlerplein 117

1082 MS Amsterdam

The Netherlands

Tel.: +31(0)20 5258579 


\title{
Associated consistency characterization of two linear values for TU games by matrix approach
}

\author{
Genjiu Xu ${ }^{\mathrm{a}, *}$, René van den Brink ${ }^{\mathrm{b}}$, Gerard van der Laan ${ }^{\mathrm{b}}$, Hao Sun ${ }^{\mathrm{a}}$ \\ ${ }^{a}$ Department of Applied Mathematics, Northwestern Polytechnical University, Xi'an, \\ Shaanxi 710072, P.R. China. \\ ${ }^{b}$ Department of Econometrics and Tinbergen Institute, $V U$ University Amsterdam, De \\ Boelelaan 1105, $1081 \mathrm{HV}$ Amsterdam, The Netherlands.
}

\begin{abstract}
Hamiache (2001) assigns to every TU game a so-called associated game and then shows that the Shapley value is characterized as the unique solution for TU games satisfying the inessential game property, continuity and associated consistency. The latter notion means that for every game the Shapley value of the associated game is equal to the Shapley value of the game itself. In this paper we show that also the EANS-value as well as the CIS-value are characterized by these three properties for appropriately modified notions of the associated game. This shows that these three values only differ with respect to the associated game. The characterization is obtained by applying the matrix approach as the pivotal technique for characterizing linear values of TU games in terms of associated consistency.
\end{abstract}

Keywords: TU games, Shapley value, EANS-value, CIS-value, associated consistency, matrix approach

\section{Introduction}

In the axiomatic analysis of cooperative games with Transferable Utility, shortly $T U$ games, consistency is an important characteristic of viable and stable solutions. A solution, or value, assigns to each TU game a vector

\footnotetext{
*Corresponding author. Tel.: +86-29-88431660, Fax: +86-29-88431657

Email addresses: xugenjiu@nwpu.edu.cn (Genjiu Xu), jrbrink@feweb.vu.nl (René van den Brink), glaan@feweb.vu.nl (Gerard van der Laan), hsun@nwpu.edu.cn (Hao Sun)
} 
whose components specify the payoffs to the players in the game. A value is consistent if it gives the same payoffs in some kind of modified game as in the original game. In the literature there are two different ideas for modifying the original game. The first one is the idea of 'reduced game', being a game that remains after some players have left and have been paid according to the prevailing value. Reduced game consistency requires that the remaining players in the reduced game receive the same payoff as in the original game. The second one is the notion of 'associated game'. In this case each coalition revalues its worth in terms of a rule related to the original game. Associated consistency requires that the value assigns the same payoff vector to the associated game as to the original game.

Reduced game consistency has been investigated for various solution concepts. It appears that various solutions satisfying reduced game consistency are only different with respect to the definition of the reduced game. Most of these results can be found in the survey papers by Driessen (1991) or Thomson (1996).

Hamiache (2001) introduced the notion of associated game and showed that the Shapley value satisfies associated game consistency. ${ }^{1}$ This consistency property of the Shapley value was studied by applying matrix analysis in Xu et al. (2008) and Hamiache (2010), respectively. Driessen (2010) generalized associated game consistency to the class of linear, symmetric and efficient values. Naumova (2009) treated a cooperative game as a bargaining problem with claim point, and studied the weighted entropy solution in terms of associated consistency. Most of these results fit within the framework of Hamiache (2001), that means that the values are characterized by the inessential game property, continuity and associated game consistency for some modified notion of associated game.

Hwang (2006) modified the definition of the associated game of Hamiache (2001) to characterize the Equal Allocation of Non-Separable costs (EANS) value, also known as the Egalitarian Non-Separable Contribution (ENSC) value in Driessen and Funaki (1991). However, this modified associated game did not allow to characterize the EANS-value by Hamiache's triple of characterizing properties. Instead Hwang (2006) used five properties to characterize

\footnotetext{
${ }^{1}$ Similar as reduced game consistency, in this paper we speak about associated game consistency instead of just associated consistency. This is also motivated because we define for different values different associated games, whereas in Hamiache (2001) only one associated game is considered to characterize the Shapley value.
} 
the EANS-value by replacing the inessential property by three other axioms namely efficiency, anonymity and translation covariance. Then it is shown that the EANS-value is characterized by these three properties, continuity and associated game consistency for the modified associated game.

The main aim of this paper is to stay within the framework of Hamiache (2001) and to characterize the EANS-value as the unique value satisfying the inessential game property, continuity and associated game consistency for an appropriately modified associated game. By applying the matrix approach, we show how to characterize linear values for TU games within the framework of Hamiache (2001). We then follow this approach to characterize the Center of gravity of Imputation Set (CIS) value, introduced in Driessen and Funaki (1991). This value is the dual value of the EANS-value. Using this duality the characterization of the EANS-value follows straightforwardly.

The paper is organized as follows. In Section 2 definitions, notations and related notions are introduced. In Section 3, we define two types of associated games and present the characterization results for the EANS and CIS-values with respect to these two associated games respectively. In Section 4 the characterization results are proved by applying the matrix approach for linear values.

\section{Definitions and notations}

A cooperative game with Transferable Utility (TU game) is a pair $\langle N, v\rangle$, where $N \subset \mathbb{N}$ is a nonempty, finite player set and $v: 2^{N} \rightarrow \mathbb{R}$ is a characteristic function on the power set of $N$, satisfying $v(\emptyset)=0$. An element $i \in N$ and a set of players $S \in 2^{N}$ are called a player and coalition respectively, and the associated real number $v(S)$ is called the worth of coalition $S$. We denote by $\mathcal{G}^{N}$ the set of all TU-games $\langle N, v\rangle$ on player set $N$ and by $\Omega=2^{N} \backslash\{\emptyset\}$ the set of all nonempty coalitions. A game $\langle N, v\rangle$ is called

inessential, if $v(S)=\sum_{i \in S} v(\{i\})$ for all $S \in \Omega$. For $\langle N, v\rangle \in \mathcal{G}^{N}$, its dual game is the game $\left\langle N, v^{D}\right\rangle$ defined by $v^{D}(S)=v(N)-v(N \backslash S)$ for all $S \in 2^{N}$. It is obvious that for an inessential game $\langle N, v\rangle$, its dual game $\left\langle N, v^{D}\right\rangle$ is given by $v^{D}(S)=v(S)$ for every $S \in 2^{N}$ and so $\left\langle N, v^{D}\right\rangle$ is inessential too. The cardinality of a finite set $K$ is denoted by $k$, so $s$ is the number of players in coalition $S$. For $K \subset \mathbb{N}$, we denote by $\mathbb{R}^{K}$ the $k$-dimensional vector space whose elements $x \in \mathbb{R}^{K}$ have components $x_{i}, i \in K$.

The main aim of cooperative game theory is to assign an allocation to every game $\langle N, v\rangle \in \mathcal{G}^{N}$, that is to assign for every game $\langle N, v\rangle$ a payoff 
$x_{i} \in \mathbb{R}$ to every $i \in N$. A single-valued solution, called value, is a function $\phi$ that assigns a single payoff vector $\phi(N, v) \in \mathbb{R}^{N}$ to every game $\langle N, v\rangle \in \mathcal{G}^{N}$. The payoff $\phi_{i}(N, v)$ of player $i$ represents an assessment by $i$ of his or her gains from participating in game $\langle N, v\rangle$. A value $\phi$ is efficient if $\sum_{i \in N} \phi_{i}(v)=$ $v(N)$ for every $\langle N, v\rangle \in \mathcal{G}^{N}$, so an efficient value divides precisely the overall earnings $v(N)$ of the grand coalition $N$ among the players in the game. The best-known efficient value is the Shapley value, denoted by $S h$. This value assigns to each $\langle N, v\rangle \in \mathcal{G}^{N}$ the payoffs

$$
S h_{i}(N, v)=\sum_{\{S \in \Omega \mid i \in S\}} \frac{(n-s) !(s-1) !}{n !}(v(S)-v(S \backslash\{i\})), \quad \text { for all } i \in N .
$$

A value $\phi$ on $\mathcal{G}^{N}$ is said to satisfy

(i) linearity, if $\phi(N, \alpha \cdot v+\beta \cdot w)=\alpha \cdot \phi(N, v)+\beta \cdot \phi(N, w)$ for all $\langle N, v\rangle$, $\langle N, w\rangle \in \mathcal{G}^{N}$, and all $\alpha, \beta \in \mathbb{R}$;

(ii) anonymity ${ }^{2}$, if $\phi_{\pi(i)}(N, \pi v)=\phi_{i}(N, v)$ for every $\langle N, v\rangle \in \mathcal{G}^{N}$, every $i \in N$, and every permutation $\pi$ on $N$;

(iii) inessential game property, if $\phi_{i}(N, v)=v(\{i\})$ for every inessential game $\langle N, v\rangle \in \mathcal{G}^{N}$, and all $i \in N$;

(iv) continuity, if for every convergent sequence of games $\left\{\left\langle N, v^{k}\right\rangle\right\}_{k=1}^{\infty}$ and its limit game $\langle N, \tilde{v}\rangle$ (i.e., for every $S \in \Omega, v^{k}(S)$ converges to $\tilde{v}(S)$ ), the corresponding sequence of payoff vectors $\left\{\phi\left(N, v^{k}\right)\right\}_{k=1}^{\infty}$ converges to the payoff vector $\phi(N, \tilde{v})$;

(v) translation covariance, if $\phi(N, v+\alpha)=\phi(N, v)+\alpha$ for every game $\langle N, v\rangle$ and every $\alpha \in \mathbb{R}^{N}$, where $\langle N, v+\alpha\rangle$ is defined by $(v+\alpha)(S)=v(S)+\sum_{i \in S} \alpha_{i}$ for all $S \in \Omega$.

It is trivial to see that any linear value $\phi$ verifies continuity.

The Equal Allocation of Non-Separable contributions (EANS) value, see Moulin (1985), assigns to each $\langle N, v\rangle \in \mathcal{G}^{N}$ the payoffs

$$
\operatorname{EANS}_{i}(N, v)=S C_{i}(N, v)+\frac{1}{n}\left[v(N)-\sum_{j \in N} S C_{j}(N, v)\right], \text { for all } i \in N
$$

where $S C_{j}(N, v)=v(N)-v(N \backslash\{j\})$ is the marginal contribution of player $j$,

\footnotetext{
${ }^{2}$ We remark that Hwang (2006) calls this symmetry although that name is usually used for the weaker property stating that $\phi_{i}(N, v)=\phi_{j}(N, v)$ whenever $i, j \in N$ are such that $v(S \cup\{i\})=v(S \cup\{j\})$ for all $S \subseteq N \backslash\{i, j\}$.
} 
$j \in N$, to the grand coalition $N{ }^{3}$ So, the EANS-value assigns to each player $i \in N$ its own marginal contribution $S C_{i}(N, v)$ and distributes the nonseparable contributions $v(N)-\sum_{j \in N} S C_{j}(N, v)$ equally among the players. Notice that $\sum_{i \in N} \operatorname{EANS}_{i}(N, v)=v(N)$ and so the EANS-value is efficient. For a game $\langle N, v\rangle \in \mathcal{G}^{N}$, the Imputation Set is the collection of all payoff vectors

$$
I(N, v)=\left\{x \in \mathbb{R}^{N} \mid \sum_{i \in N} x_{i}=v(N) \text { and } x_{i} \geq v(\{i\}) \text { for all } i \in N\right\} .
$$

The Center of gravity of the Imputation Set (CIS) value, introduced by Driessen and Funaki (1991), is consequently defined as

$$
C I S_{i}(N, v)=v(\{i\})+\frac{1}{n}\left[v(N)-\sum_{j \in N} v(\{j\})\right], \text { for all } i \in N
$$

Obviously, also the CIS-value is efficient. Since, by definition of the dual game, $v^{D}(\{j\})=v(N)-v(N \backslash\{j\})=S C_{j}(N, v)$ for all $j \in N$, it follows that $\operatorname{EANS}(N, v)=C I S\left(N, v^{D}\right)$, so the EANS-value and the CIS value are each other's dual. On the other hand, the Shapley value is self-dual, i.e. $S h(N, v)=S h\left(N, v^{D}\right)$. Finally, notice that both values are linear as well as continuous.

For a class of equal surplus sharing solutions, including both the EANSvalue and the CIS-value, van den Brink and Funaki (2009) provided characterizations in terms of some type of reduced game consistency. In this paper, we follow Hamiache's approach and provide an axiomatization of the EANS and CIS-value by associated game consistency.

\section{Associated consistency for the EANS and CIS-values}

To characterize the Shapley value, Hamiache (2001) introduced a consistency axiom with respect to a specific associated game. For a game $\langle N, v\rangle$, an associated game is a game $\left\langle N, v^{A}\right\rangle$ that revalues for every coalition $S \in \Omega$ its worth from $v(S)$ to a worth $v^{A}(S)$ related to the original game according to some function on the class of games $\mathcal{G}^{N}$, i.e., there is some function $f$

\footnotetext{
${ }^{3}$ The EANS-value has been introduced originally for costs games (i.e. for each $S, v(S)$ are the costs of coalition $S$ instead of revenues) as the EANS cost solution. For costs games $S C_{j}(N, v)$ are the separable costs of player $j$.
} 
on $\mathcal{G}^{N}$ that maps $\langle N, v\rangle$ into $\langle N, f(v)\rangle$. Hamiache's associated consistency axiom, in this paper renamed as associated game consistency is defined with reference to an associated game rule.

Axiom 1. For a rule $f: \mathcal{G}^{N} \rightarrow \mathcal{G}^{N}$, a value $\phi$ satisfies $f$-associated game consistency if $\phi(N, v)=\phi(N, f(v))$ for every game $\langle N, v\rangle \in \mathcal{G}^{N}$.

In the sequel we will often just speak about associated game consistency when there is no confusion about the rule $f$. According to associated game consistency, a value behaves invariant under the adaptation of the game into the associated game. To characterize the Shapley value, Hamiache (2001) defined for some value $\lambda, 0 \leq \lambda \leq 1$, the associated game, denoted by $\left\langle N, v_{\lambda}^{S h}\right\rangle$, as

$$
v_{\lambda}^{S h}(S)=v(S)+\lambda \sum_{j \in N \backslash S}[v(S \cup\{j\})-v(S)-v(\{j\})], \text { for all } S \in \Omega .
$$

For an interpretation we refer to Hamiache (2001). Briefly, coalition $S$ can earn $v(S)$ on its own. Knowing that eventually the 'grand coalition' $N$ will be formed, coalition $S$ will earn somewhat more or less than this worth. The associated game specifies what coalition $S$ expects to earn additional to its worth (where this addition can be negative). The different associated games differ with respect to what is the additional earnings coalition $S$ expects above its own worth. In the associated game of Hamiache this is a fraction of all the surplusses that can be generated by bilateral cooperation between $S$ and each player outside $S$.

The Shapley value is characterized as the unique solution that satisfies associated game consistency with respect to $\left\langle N, v_{\lambda}^{S h}\right\rangle$ for $0<\lambda<\frac{2}{n}$, continuity and the inessential game property. To show this, Hamiache (2001) considered the infinite sequence of games $\left\{\left\langle N, v^{k, S h}\right\rangle\right\}_{k=1}^{\infty}$, where, for some fixed value of $\lambda, 0<\lambda<\frac{2}{n}, v^{1, S h}=v_{\lambda}^{S h}$ and $v^{k+1, S h}=\left(v^{k, S h}\right)_{\lambda}^{S h}, k=1,2, \ldots$, i.e., $v^{k+1, S h}$ is the associated game to $v^{k, S h}$ according to the given rule. It is then proved that this sequence of repeated associated games converges to the inessential limit game $\langle N, \widetilde{v}\rangle$ given by

$$
\widetilde{v}(S)=\sum_{i \in S} S h_{i}(N, v)
$$

Now, let $\phi$ be a solution satisfying associated game consistency with respect to $\left\langle N, v_{\lambda}^{S h}\right\rangle$, continuity and the inessential game property. Then by the first 
two properties we have that

$$
\phi(N, v)=\phi\left(N, v^{1, S h}\right)=\cdots=\phi\left(N, v^{k, S h}\right)=\cdots=\phi(N, \widetilde{v})
$$

and with the inessential game property it then follows that $\phi_{i}(N, v)=S h_{i}(N, v)$, $i \in N$.

To characterize the EANS-value Hwang (2006) defined for some value $\lambda$, the associated game, denoted by $\left\langle N, v_{\lambda}^{H w}\right\rangle$, as

$$
v_{\lambda}^{H w}(S)=v(S)+\lambda \sum_{j \in N \backslash S}\left[v(S \cup\{j\})-v(S)-S C_{j}(N, v)\right] \text {, for all } S \in \Omega \text {. }
$$

Compared to Hamiache's associated game, the term $v(\{j\})$ in the definition of $\left\langle N, v_{\lambda}^{S h}\right\rangle$ is replaced by its dual worth $S C_{j}(N, v)$ in $\left\langle N, v_{\lambda}^{H w}\right\rangle$. This seems somewhat surprising since the Shapley value is not the dual solution of the EANS-value. In fact, the dual of the EANS-value is the CIS-value while the Shapley value is self-dual. However, later in this paper we will see that this has to do with the different frameworks where the two associated games are applied, whereas we introduce an associated game for the EANS-value in the framework of Hamiache (2001) (and consequently also obtain an associated game for its dual, the CIS-value).

Now, the infinite sequence of repeated associated games $\left\{\left\langle N, v^{k, H w}\right\rangle\right\}_{k=1}^{\infty}$, where, for some fixed value of $\lambda, 0<\lambda<\frac{2}{n-1}, v^{1, H} w=v_{\lambda}^{H w}$ and $v^{k+1, H w}=$ $\left(v^{k, H w}\right)_{\lambda}^{H w}, k=1,2, \ldots$, converges to the limit game $\langle N, \widehat{v}\rangle$ given by

$$
\widehat{v}(S)=\sum_{j \in S} S C_{j}(N, v)+\left[v(N)-\sum_{j \in N} S C_{j}(N, v)\right], \text { for all } S \in \Omega .
$$

This limit game $\langle N, \widehat{v}\rangle$ is not inessential, but is the sum of the inessential game $\langle N, v\rangle$ given by $v(S)=\sum_{j \in S} S C_{j}(N, v), S \in \Omega$, and the constant game $\langle N, v\rangle$ given by $v(S)=v(N)-\sum_{j \in N} S C_{j}(N, v), S \in \Omega$. As a consequence, the inessential game property can not be used now to characterize the EANS-value. Instead Hwang (2006) shows that the EANS-value is characterized as the unique solution satisfying associated game consistency with respect to $\left\langle N, v_{\lambda}^{H w}\right\rangle$ (for $0<\lambda<\frac{2}{n-1}$ ), continuity and the three axioms of efficiency, anonymity and translation covariance. So, Hwang's approach does not fit within Hamiache's framework in the sense that the limit game is not inessential and consequently in the corresponding axiom system the inessential property is replaced by efficiency, anonymity and translation covariance. 
In the sequel we establish a characterization of the EANS-value within the framework of Hamiache (2001). So, we define an associated game in such a way that the EANS-value is the unique solution satisfying associated game consistency with respect to this game, continuity and the inessential game property. For some value $\lambda, 0<\lambda<1$, the associated game $\left\langle N, v_{\lambda}^{E}\right\rangle$ is defined as, for all $S \in \Omega$

$$
v_{\lambda}^{E}(S)=v(S)+\lambda\left[\frac{s}{n}\left(v(N)-\sum_{j \in N} S C_{j}(N, v)\right)-\left(v(S)-\sum_{j \in S} S C_{j}(N, v)\right)\right] .
$$

This associated game can been interpreted as follows. Every coalition $S$ assumes that, besides it worth $v(S)$, the nonspearable costs are allocated over the players. If the nonseparable costs are allocated equally over the players in $N$ than each player gets $\frac{1}{n}\left(v(N)-\sum_{j \in N} S C_{j}(N, v)\right)$ of it. So, coalition $S$ would receive $\frac{s}{n}\left(v(N)-\sum_{j \in N} S C_{j}(N, v)\right)$ of this. But since coalition $S$ already assumes to get $v(S)$, it should get less of the nonseparable cost(if $v(S)$ is positive). How much coalition $S$ gets from the nonseparable costs is determined by the difference between the nonseparable costs $v(S)-$ $\sum_{j \in S} S C_{j}(N, v)$ within $S$ and the share of $S$ in case the nonseparable costs of $N$ would be allocated equally among all players.

We will show in Section 4 that the EANS-value satisfies associated game consistency for the game $\left\langle N, v_{\lambda}^{E}\right\rangle$, thus $\operatorname{EAN} S_{i}(N, v)=E A N S_{i}\left(N, v_{\lambda}^{E}\right), i \in N$, for every game $\langle N, v\rangle \in \mathcal{G}^{N}$. We then consider the infinite sequence of repeated associated games $\left\{\left\langle N, v^{k, E}\right\rangle\right\}_{k=1}^{\infty}$, where, for some fixed value of $\lambda$, $v^{1, E}=v_{\lambda}^{E}$ and $v^{k+1, E}=\left(v^{k, E}\right)_{\lambda}^{E}, k=1,2, \ldots$, and show that this sequence converges to the inessential limit game $\left\langle N, v^{*}\right\rangle$ given by

$$
v^{*}(S)=\sum_{j \in S} \operatorname{EANS}_{j}(N, v), \text { for all } S \in \Omega .
$$

It then follows that, in accordance with the framework of Hamiache, the EANS-value is characterized by the associated game consistency with respect to $\left\langle N, v^{E}\right\rangle$, continuity and the inessential game property. This yields the following theorem, that will be proven in Section 4 .

Theorem 3.1. The EANS-value is the unique value satisfying associated game consistency with respect to $\left\langle N, v_{\lambda}^{E}\right\rangle$, continuity and the inessential game property. 
Motivated by the dual relationship between the CIS-value and the EANSvalue, replacing in $\left\langle N, v_{\lambda}^{E}\right\rangle$ the term $S C_{j}(N, v)=v^{D}(\{j\})$ by its dual worth $v(\{j\})$, we define for $\lambda, 0<\lambda<1$, the associated game $\left\langle N, v_{\lambda}^{C}\right\rangle$ as, for all $S \in \Omega$,

$$
v_{\lambda}^{C}(S)=v(S)+\lambda\left[\frac{s}{n}\left(v(N)-\sum_{j \in N} v(\{j\})\right)-\left(v(S)-\sum_{j \in S} v(\{j\})\right)\right] .
$$

The next proposition shows that for every game $\langle N, v\rangle$, the dual game of its associated game (3.1) is the associated game (3.2) of its dual game $\left\langle N, v^{D}\right\rangle$.

Proposition 3.2. For any game $\langle N, v\rangle \in \mathcal{G}^{N},\left\langle N,\left(v_{\lambda}^{E}\right)^{D}\right\rangle=\left\langle N,\left(v^{D}\right)_{\lambda}^{C}\right\rangle$.

Proof. For any game $\langle N, v\rangle \in \mathcal{G}^{N}$, it is obvious that $v_{\lambda}^{E}(N)=v(N)$ and $v_{\lambda}^{C}(N)=v(N)$ by (3.1) and (3.2). Further, for all $S \in \Omega$, we have

$$
\begin{aligned}
\left(v_{\lambda}^{E}\right)^{D}(S) & =v_{\lambda}^{E}(N)-v_{\lambda}^{E}(N \backslash S) \\
= & v(N)-v(N \backslash S) \\
& -\lambda\left[\frac{n-s}{n}\left(v(N)-\sum_{j \in N} S C_{j}(N, v)\right)-\left(v(N \backslash S)-\sum_{j \in N \backslash S} S C_{j}(N, v)\right)\right] \\
= & v(N)-v(N \backslash S)+\lambda\left[\frac{s}{n}\left(v(N)-\sum_{j \in N} S C_{j}(N, v)\right)\right. \\
& \left.\quad-v(N)+\sum_{j \in N} S C_{j}(N, v)+v(N \backslash S)-\sum_{j \in N \backslash S} S C_{j}(N, v)\right] \\
= & v(N)-v(N \backslash S) \\
& +\lambda\left[\frac{s}{n}\left(v(N)-\sum_{j \in N} S C_{j}(N, v)\right)-\left(v(N)-v(N \backslash S)-\sum_{j \in S} S C_{j}(N, v)\right)\right] .
\end{aligned}
$$

On the other hand, since $v^{D}(\{j\})=S C_{j}(N, v)$ for all $i \in N$, we have

$$
\begin{aligned}
\left(v^{D}\right)_{\lambda}^{C}(S) & =v^{D}(S)+\lambda\left[\frac{s}{n}\left(v^{D}(N)-\sum_{j \in N} v^{D}(\{j\})\right)-\left(v^{D}(S)-\sum_{j \in S} v^{D}(\{j\})\right)\right] \\
= & v(N)-v(N \backslash S) \\
& +\lambda\left[\frac{s}{n}\left(v(N)-\sum_{j \in N} S C_{j}(N, v)\right)-\left(v(N)-v(N \backslash S)-\sum_{j \in S} S C_{j}(N, v)\right)\right] .
\end{aligned}
$$

We conclude that $\left\langle N,\left(v_{\lambda}^{E}\right)^{D}\right\rangle=\left\langle N,\left(v^{D}\right)_{\lambda}^{C}\right\rangle$. 
Taking again the infinite sequence $\left\{\left\langle N, v^{k, C}\right\rangle\right\}_{k=1}^{\infty}$, where, for some fixed value of $\lambda, v^{1, C}=v_{\lambda}^{C}$ and $v^{k+1, C}=\left(v^{k, C}\right)_{\lambda}^{C}, k=1,2, \ldots$, also the CISvalue is characterized in terms of the axiom system within the framework of Hamiache (2001).

Theorem 3.3. The CIS-value is the unique value satisfying associated game consistency with respect to $\left\langle N, v_{\lambda}^{C}\right\rangle$, continuity and the inessential game property.

In the next section we first prove Theorem 3.3 by using the matrix approach. Then the characterization of the EANS-value in Theorem 3.1 is obtained immediately from the relationship given in Proposition 3.2.

\section{Matrix approach to associated consistency for linear values}

As is well-known, every game $\langle N, v\rangle \in \mathcal{G}^{N}$ can be identified by a column vector in the $\left(2^{n}-1\right)$-dimensional vector space $\mathbb{R}^{2^{n}-1}$, where the components of such a vector represent the worths $v(S)$ of the $2^{n}-1$ nonempty coalitions in $\Omega .{ }^{4}$ We assume that the coalitions are ordered according to the lexicographical ordering. Obviously, any linear operator on the game space $\mathcal{G}^{N}$ has a matrix interpretation, in particular a linear value $\phi$ can be written as $\phi(N, v)=M^{\phi} \cdot v$ with $M^{\phi}$ an $n \times\left(2^{n}-1\right)$ matrix and any type of associated game $\left\langle N, v^{A}\right\rangle$ as $v^{A}=M^{A} \cdot v$ with $M^{A}$ a square matrix of size $2^{n}-1$.

The algebraic representation and matrix approach to cooperative game theory have appeared to be natural as well as powerful. Kleinberg and Weiss (1985) constructed a direct sum decomposition of the null space and studied equivalent classes of games with respect of the Shapley value, i.e., two games are in the same class if they have the same Shapley value. Dragan (1991, 1996) introduced the potential basis to study the weighted Shapley value and the Banzhaf value. Grabisch (2000) presented a way to use matrices instead of operators to study linear nonsingular functions on the class of cooperative games. Recently, Hernandez-Lamoneda et al. (2007) introduced a natural representation theory by computing a direct sum decomposition for the game space $\mathcal{G}^{N}$. Following this scheme, well known results as well as new theorems and characterizations of a certain class of linear symmetric values are derived. The matrix approach was applied to study associated game consistency for

\footnotetext{
${ }^{4}$ The worth of the empty set is not represented, since $v(\emptyset)=0$ for every $\langle N, v\rangle \in \mathcal{G}^{N}$.
} 
the Shapley value by $\mathrm{Xu}$ et al. (2008) and Hamiache (2010), respectively. Recently, this approach has also been applied by Hamiache (2012) to study the efficient Aumann-Drèze value for TU games with coalition structure and the efficient Myerson value for TU games with communication structure.

$\mathrm{Xu}$ et al. (2008) introduced the notion of coalitional matrices representing linear operators on the game space $\mathcal{G}^{N}$. A matrix $M$ is called row (column)coalitional if the number of rows (respectively columns) is $2^{n}-1$ and are indexed in lexicographic ordering by all coalitions $S \in \Omega$. A matrix $M$ is called square-coalitional if it is both row-coalitional and column-coalitional. Then the following results are shown.

(i) A value $\phi$ is linear on $\mathcal{G}^{N}$ if and only if there is a column-coalitional matrix $M^{\phi}$ such that $\phi(N, v)=M^{\phi} \cdot v$ for any game $\langle N, v\rangle \in \mathcal{G}^{N}$.

(ii) For any linear operator that maps game $\langle N, v\rangle$ in its associated game $\left\langle N, v_{\lambda}\right\rangle$ for some value $\lambda$, and the corresponding infinite sequence of repeated associated games $\left\{\left\langle N, v^{k}\right\rangle\right\}_{k=1}^{\infty}$ with $v^{1}=v_{\lambda}$ and $v^{k+1}=\left(v^{k}\right)_{\lambda}, k=1,2, \ldots$, there is an associated square-coalitional matrix $M_{\lambda}$, such that $v_{\lambda}=M_{\lambda} \cdot v$ and $v^{k}=\left(M_{\lambda}\right)^{k} \cdot v, k=1,2, \ldots$

(iii) When the sequence of associated games converges to limit game $\langle N, \tilde{v}\rangle$, then $\tilde{v}=\tilde{M} \cdot v$ with $\tilde{M}=\lim _{k \rightarrow \infty}\left(M_{\lambda}\right)^{k}$.

Applying these results the matrix approach can now be used to characterize values within the axiom system of Hamiache (2001).

First, the associated game consistency of a linear value $\phi$ with respect to the associated game $\left\langle N, v_{\lambda}\right\rangle$ follows from $M^{\phi}$ being invariant under multiplication with the associated matrix $M_{\lambda}$, i.e., $M^{\phi}=M^{\phi} M_{\lambda}$.

Second, using the diagonal decomposition $M_{\lambda}=P D_{\lambda} P^{-1}$, where $D_{\lambda}$ is the diagonal matrix with all diagonal elements being the eigenvalues of $M_{\lambda}$ and $P$ is the matrix consisting of all corresponding eigenvectors, it follows that $\left(M_{\lambda}\right)^{k}=P\left(D_{\lambda}\right)^{k} P$. By the restrictions on the value of $\lambda$, it follows that all eigenvalues of $M_{\lambda}$ are in $[0,1]$. It then follows that $\lim _{k \rightarrow \infty}\left(D_{\lambda}\right)^{k}$ exists and SO

$$
\tilde{M}=\lim _{k \rightarrow \infty}\left(M_{\lambda}\right)^{k}=P \lim _{k \rightarrow \infty}\left(D_{\lambda}\right)^{k} P^{-1}=P D P^{-1} \text {. }
$$

Applying the continuity property yields $\phi(N, v)=\tilde{M} \cdot v$.

Third, to apply the inessential game property it is required that $M_{\lambda}$ has an eigenvector equal to one (otherwise $\lim _{k \rightarrow \infty}\left(D_{\lambda}\right)^{k}$ is the zero matrix) and that $\tilde{M}$ is a row-inessential square-coalitional matrix, i.e., the row of $\tilde{M}$ indexed 
by a coalition $S \in \Omega$ is equal to the sum over all $i \in S$ of the rows of $\tilde{M}$ indexed by $i$. Thus $\tilde{M}_{S}=\sum_{i \in S} \tilde{M}_{i}$ where, in this paper, the index of the row with respect to a singleton coalition $\{i\}, i \in N$, is shortened as $i$.

In the following we apply this matrix technique to prove Theorems 3.1 and 3.3. Since the comparatively simple form of the CIS-value simplifies the proof, we first prove the latter theorem and then we use duality between the CIS and EANS-value to prove the first one. However, notice that the characterizing procedures of these two values are equivalent from an algebraic point of view.

For any game $\langle N, v\rangle$, the CIS-value can be written in matrix form as

$$
C I S(N, v)=M^{C} \cdot v,
$$

with the element $\left[M^{C}\right]_{i, S}$ on the $i$-th row and the column indexed by $S \in \Omega$ given by

$$
\left[M^{C}\right]_{i, S}= \begin{cases}\frac{1}{n}, & \text { if } S=N ; \\ 1-\frac{1}{n}, & \text { if } S=\{i\} ; \\ -\frac{1}{n}, & \text { if } S=\{j\}, j \in N \backslash\{i\} ; \\ 0, & \text { otherwise. }\end{cases}
$$

Further, rewriting the worth $v_{\lambda}^{C}(S), S \in \Omega$, of the associated game given in equation (3.2) as

$$
v_{\lambda}^{C}(S)=(1-\lambda) v(S)+\frac{s}{n} \lambda v(N)-\frac{s}{n} \lambda \sum_{j \in N \backslash S} v(\{j\})+\left(1-\frac{s}{n}\right) \lambda \sum_{j \in S} v(\{j\}),
$$

the associated game $\left\langle N, v_{\lambda}^{C}\right\rangle$ can be written as

$$
v_{\lambda}^{C}=M_{\lambda}^{C} \cdot v,
$$

with the element $\left[M_{\lambda}^{C}\right]_{S \in \Omega, T \in \Omega}$ given by

$$
\left[M_{\lambda}^{C}\right]_{S, T}= \begin{cases}1-\frac{1}{n} \lambda, & \text { if } T=S, s=1 \\ 1-\lambda, & \text { if } T=S, 2<s<n \\ 1, & \text { if } T=S, s=n ; \\ \frac{s}{n} \lambda, & \text { if } T \neq S, t=n ; \\ -\frac{s}{n} \lambda, & \text { if } T=\{j\}, j \notin S, 1 \leq s \leq n \\ \left(1-\frac{s}{n}\right) \lambda, & \text { if } T=\{j\}, j \in S, 2<s<n \\ 0, & \text { otherwise. }\end{cases}
$$


In the next proposition the associated game consistency for the CIS-value with respect to the associated game $\left\langle N, v_{\lambda}^{C}\right\rangle$ is stated and proved in terms of the matrix approach. For notational convenience we often write $i$ instead of $\{i\}$.

Proposition 4.1. The matrix $M^{C}$ is invariant under multiplication with the associated matrix $M_{\lambda}^{C}$, that is $M^{C}=M^{C} M_{\lambda}^{C}$.

Proof. We check the matrix equality by showing $\left[M^{C} M_{\lambda}^{C}\right]_{i, S}=\left[M^{C}\right]_{i, S}$ for all $i \in N$ and all $S \in \Omega$. For all $i \in N$ and all $S \in \Omega$, we have

$$
\begin{aligned}
& {\left[M^{C} M_{\lambda}^{C}\right]_{i, S}=\sum_{T \in \Omega}\left[M^{C}\right]_{i, T}\left[M_{\lambda}^{C}\right]_{T, S}} \\
& \quad=\left[M^{C}\right]_{i, i}\left[M_{\lambda}^{C}\right]_{i, S}+\sum_{j \neq i}\left[M^{C}\right]_{i, j}\left[M_{\lambda}^{C}\right]_{j, S}+\left[M^{C}\right]_{i, N}\left[M_{\lambda}^{C}\right]_{N, S} .
\end{aligned}
$$

From (4.2) and (4.5), if $S=N$, then (4.6) yields

$$
\left[M^{C} M_{\lambda}^{C}\right]_{i, N}=\left(1-\frac{1}{n}\right) \cdot \frac{1}{n} \lambda-(n-1) \frac{1}{n} \cdot \frac{1}{n} \lambda+\frac{1}{n} \cdot 1=\frac{1}{n}=\left[M^{C}\right]_{i, N} .
$$

Similarly, using (4.2), (4.5) and (4.6) yields that $\left[M^{C} M_{\lambda}^{C}\right]_{i, S}=\left[M^{C}\right]_{i, S}$ for all $i \in N$ and all $S \neq N$.

In the following we investigate the diagonalizable decomposition of $M_{\lambda}^{C}$ by analyzing its eigenvalues and eigenvectors. A $\left(2^{n}-1\right)$-dimensional vector $x$ with components $x_{S}$ indexed by the coalitions $S \in \Omega$ is row-inessential if $x_{S}=\sum_{i \in S} x_{i}$ for every $S \in \Omega$.

Lemma 4.2. For the associated matrix $M_{\lambda}^{C}$, we have

1. 1 is an eigenvalue of $M_{\lambda}^{C}$, the eigenvectors corresponding to eigenvalue 1 are row-inessential and the dimension of the corresponding eigenspace is equal to $n$;

2. $1-\lambda$ is an eigenvalue of $M_{\lambda}^{C}$, and the $\operatorname{rank} R\left[M_{\lambda}^{C}-(1-\lambda) I\right] \leq n$;

3. the matrix $M_{\lambda}^{C}$ is diagonalizable.

Proof. 1. Let $I$ be the identity matrix. Since $v_{\lambda}^{C}(N)=v(N)$, the last row of matrix $M_{\lambda}^{C}-I$ is the zero vector. So 1 is an eigenvalue of $M_{\lambda}^{C}$. Let the $\left(2^{n}-1\right)$-dimensional vector $x$ be an eigenvector corresponding to eigenvalue 
1 with components $x_{S}, S \in \Omega$. Since $\left(M_{\lambda}^{C}-I\right) x=\mathbf{0}$ and $\lambda \neq 0$, we have for all $S \in \Omega$ with $1 \leq s<n$ that

$$
-x_{S}+\frac{s}{n} x_{N}-\frac{s}{n} \sum_{j \notin S} x_{j}+\left(1-\frac{s}{n}\right) \sum_{j \in S} x_{j}=0 .
$$

For $s=1$, i.e. $S=\{j\}, j \in N$, this yields that

$$
-\frac{1}{n} x_{j}+\frac{1}{n} x_{N}-\frac{1}{n} \sum_{j \notin S} x_{j}=0 .
$$

That is $x_{N}=\sum_{j \in N} x_{j}$. Taking this into (4.7), we have for all $S \in \Omega, 2 \leq$ $s<n$,

$$
-x_{S}+\frac{s}{n} \sum_{j \in S} x_{j}+\left(1-\frac{s}{n}\right) \sum_{j \in S} x_{j}=0 .
$$

So, we conclude that

$$
x_{S}=\sum_{j \in S} x_{j}, \text { for all } S \in \Omega,
$$

and thus any eigenvector $x$ corresponding to eigenvalue 1 is row-inessential. From this it follows that the dimension of the corresponding eigenspace is equal to $n$.

2. Let $A=M_{\lambda}^{C}-(1-\lambda) I$. Considering the columns of matrix $A$, denote by $[A]_{. T}$ the column of $A$ indexed by coalition $T \in \Omega$. Obviously, all columns $[A]_{\text {.T }}$ with $2 \leq t \leq n-1$ are zero vectors 0 . This implies that $1-\lambda$ is an eigenvalue of $M_{\lambda}^{C}$. It is left to analyze the $n+1$ non-zero columns $[A]_{T_{T}}$ for the $n$ singleton coalitions $T=\{j\}$ and the grand coalition $T=N$. It turns out that $\sum_{j \in N}[A]_{. j}=\mathbf{0}$, i.e., the $n$ columns indexed by the singleton coalitions sum up to the zero vector. To show this, consider $S \in \Omega$. If $S=\{i\}, i \in N$, then

$$
\sum_{j \in N}[A]_{i, j}=[A]_{j, j}+\sum_{j \neq i}[A]_{i, j}=\lambda\left(1-\frac{1}{n}\right)+\lambda\left(-\frac{1}{n}\right) \cdot(n-1)=0 .
$$

Further, if $S \in \Omega$ is a coalition with at least two members, $2 \leq s<n$, it follows that

$$
\sum_{j \in N}[A]_{S, j}=\sum_{j \in S}[A]_{S, j}+\sum_{j \notin S}[A]_{S, j}=\lambda\left(1-\frac{s}{n}\right) \cdot s+\lambda\left(-\frac{s}{n}\right) \cdot(n-s)=0 .
$$


If $s=n$, since $[A]_{N, j}=0$ for all $j \in N$, so $\sum_{j \in N}[A]_{N, j}=0$.

So, the sum over all $j \in N$ of the columns $[A]_{. j}$ is equal to the zero vector $\mathbf{0}$ and therefore these columns are linear dependent. Together with the fact that all columns $[A]_{T}$ with $2 \leq t \leq n-1$ are zero vectors, it follows that $R\left[M_{\lambda}^{C}-(1-\lambda) I\right] \leq n$.

3. Denote by $m_{1}, m_{1-\lambda}$ the multiplicities of the eigenvalues $1,1-\lambda$ respectively, and by $d_{1}, d_{1-\lambda}$ the dimensions of the corresponding eigenspaces. Since the order of $M_{\lambda}^{C}$ is $2^{n}-1$, the dimension $d_{1-\lambda}$ of the solution space of the linear system $\left[M_{\lambda}^{C}-(1-\lambda) I\right] x=\mathbf{0}$ satisfies $d_{1-\lambda}=2^{n}-1-R\left[M_{\lambda}^{C}-(1-\lambda) I\right] \geq$ $2^{n}-1-n$. From the theory of linear algebra it is known that the sum of the multiplicities of all eigenvalues of a matrix equals its order and each multiplicity is at least the dimension of the corresponding eigenspace. From the facts 1 and 2 proved above we know that 1 and $1-\lambda$ are eigenvalues of $M_{\lambda}^{C}$ and $d_{1}=n$. It follows that

$$
2^{n}-1 \geq m_{1}+m_{1-\lambda} \geq d_{1}+d_{1-\lambda} \geq n+2^{n}-1-n=2^{n}-1,
$$

which can only be true if all inequalities hold with equalities and thus $m_{1-\lambda}=$ $d_{1-\lambda}=2^{n}-1-n$. So $M_{\lambda}^{C}$ has no other eigenvalues and is diagonalizable.

To prove that for given $\lambda, 0<\lambda<1$ the infinite sequence of repeated associated games $\left\{\left\langle N, v^{k, C}\right\rangle\right\}_{k=1}^{\infty}$ converges to an inessential game, we use the results of the lemma above and the results given in next lemma proved in Xu et al. (2008).

Lemma 4.3 (Xu et al. (2008)). For $N \subset \mathbb{N}$ and $m \in \mathbb{N}$, let $M$ be a $\left(2^{n}-\right.$ 1) $\times m$ row-coalitional matrix and $A$ be a matrix with $m$ rows.

1. If $M$ is inessential, then the row-coalitional matrix $M A$ is inessential.

2. If $A$ is invertible, then $M A$ is inessential if and only if $M$ is inessential.

3. For every game $\langle N, v\rangle \in \mathcal{G}^{N}$, if $M$ is inessential, then $\langle N, M \cdot v\rangle$ is inessential.

Theorem 4.4. For any game $\langle N, v\rangle \in \mathcal{G}^{N}$ and fixed $\lambda, 0<\lambda<1$, the sequence of repeated associated games $\left\{\left\langle N, v^{k, C}\right\}_{k=1}^{\infty}\right.$ converges and the limit game is inessential.

Proof. By 3 of Lemma 4.2, the matrix $M_{\lambda}^{C}$ is diagonalizable. Denote $M_{\lambda}^{C}=$ $P D_{\lambda}^{C} P^{-1}$, where $D_{\lambda}^{C}=\operatorname{diag}(1, \cdots, 1,1-\lambda, \cdots, 1-\lambda)$ and $P$ consists of 
eigenvectors of $M_{\lambda}^{C}$ corresponding to eigenvalues 1 and $1-\lambda$. Since $0<\lambda<1$, we have

$$
\lim _{k \rightarrow \infty}\left(M_{\lambda}^{C}\right)^{k}=\lim _{k \rightarrow \infty} P\left(D_{\lambda}^{C}\right)^{k} P^{-1}=P \lim _{k \rightarrow \infty}\left(D_{\lambda}^{C}\right)^{k} P^{-1}=P D P^{-1},
$$

with $D=\operatorname{diag}(1, \cdots, 1,0, \cdots, 0)$ and the elements 1 repeat $n$ times. For the row-coalitional matrix $P D$ we have

$$
P D=\left[x^{1}, x^{2}, \cdots, x^{n}, \mathbf{0}, \cdots, \mathbf{0}\right],
$$

where the column vectors $x^{i}(i=1,2, \ldots, n)$ are different eigenvectors of $M_{\lambda}^{C}$ corresponding to eigenvalue 1 . By 1 of Lemma 4.2 these eigenvectors are all inessential vectors. Therefore $P D$ is inessential and by 2 of Lemma 4.3 also $P D P^{-1}$ is inessential. By the matrix representation of $\left\{\left\langle N, v^{k, C}\right\rangle\right\}_{k=1}^{\infty}$, we have

$$
\lim _{k \rightarrow \infty} v^{k, C}=\lim _{k \rightarrow \infty}\left(M_{\lambda}^{C}\right)^{k} \cdot v=P D P^{-1} \cdot v .
$$

By 3 of Lemma 4.3 , game $\left\langle N, P D P^{-1} \cdot v\right\rangle$ is inessential.

We are now ready to prove Theorem 3.3.

Proof of Theorem 3.3. By Proposition 4.1 the CIS-value satisfies the associated game consistency with respect to $\left\langle N, v_{\lambda}^{C}\right\rangle$. Further it is straightforward to see that the CIS-value satisfies continuity and the inessential game property.

Next, let $\phi$ be a value satisfying these three axioms. For any game $\langle N, v\rangle$, we show that $\phi(N, v)=C I S(N, v)$. According to Theorem 4.4 the sequence of repeated associated games $\left\{\left\langle N, v^{k, C}\right\rangle\right\}_{k=1}^{\infty}$ converges to an inessential game. Denote the limit game by $\left\langle N, v^{* *}\right\rangle$. By associated game consistency and continuity of $\phi$, it holds that $\phi(N, v)=\phi\left(N, v^{* *}\right)$. Further the inessential game property yields $\phi_{i}\left(N, v^{* *}\right)=v^{* *}(\{i\})$ for all $i \in N$. So, $\phi$ is uniquely determined by the three axioms. Since the CIS-value satisfies these three axioms, it follows $\phi(N, v)=C I S(N, v)$.

It remains to prove Theorem 3.1 characterizing the EANS-value. Therefore, recall from Proposition 3.2 that the dual game of the associated game (3.1) of $v$ is the associated game (3.2) of the dual game of $v$. In terms of similarity of matrices, $\mathrm{Xu}$ et al. (2009) derived a similar type of duality associated game for the Shapley value, based on the dual operator $Q$ on the 
game space. So $Q$ is a square-coalitional matrix with $Q^{-1}=Q$ that maps any game $\langle N, v\rangle$ into its dual game $\left\langle N, v^{D}\right\rangle$, that is $v^{D}=Q \cdot v$, where the dual matrix $Q=[Q]_{S \in \Omega, T \in \Omega}$ is given by

$$
[Q]_{S, T}= \begin{cases}-1, & \text { if } T=N \backslash S \text { and } S \neq N \\ 1, & \text { if } T=N \\ 0, & \text { otherwise }\end{cases}
$$

Similar as for the CIS-value, for any game $\langle N, v\rangle$, the EANS-value can be written in matrix form as $\operatorname{EANS}(N, v)=M^{E} \cdot v$ and correspondingly the associated game $\left\langle N, v_{\lambda}^{E}\right\rangle$ can be written as

$$
v_{\lambda}^{E}=M_{\lambda}^{E} \cdot v
$$

with $M_{\lambda}^{E}$ the square-coalitional matrix reflecting (3.1). Since $\operatorname{EANS}(v)=$ $C I S\left(v^{D}\right)$, we obtain from the CIS-value matrix representation (4.1) that

$$
M^{E}=M^{C} Q \text {, or equivalently } M^{C}=M^{E} Q .
$$

Also in the next proposition, we restate the relationship between the associated game $\left\langle N, v_{\lambda}^{E}\right\rangle$ of the EANS-value and the associated game $\left\langle N, v_{\lambda}^{C}\right\rangle$ of the CIS-value in terms of the corresponding transformation matrices, by the dual operator $Q$.

Proposition 4.5. Let $M_{\lambda}^{E}$ and $M_{\lambda}^{C}$ be the square-coalitional matrices introduced in (4.4) and (4.8) for the associated games (3.1) and (3.2) respectively. Then

$$
M_{\lambda}^{E}=Q M_{\lambda}^{C} Q, \text { or equivalently } M_{\lambda}^{C}=Q M_{\lambda}^{E} Q
$$

Proof. For any game $\langle N, v\rangle$ we have by Proposition 3.2 that $\left\langle N,\left(v_{\lambda}^{E}\right)^{D}\right\rangle=$ $\left\langle N,\left(v^{D}\right)_{\lambda}^{C}\right\rangle$. In matrix representation this yields

$$
Q \cdot v_{\lambda}^{E}=M_{\lambda}^{C} \cdot v^{D}, \text { or } Q M_{\lambda}^{E} \cdot v=M_{\lambda}^{C} Q \cdot v
$$

By the arbitrariness of $\langle N, v\rangle$, we get $Q M_{\lambda}^{E}=M_{\lambda}^{C} Q$. Since $Q^{-1}=Q$, we get by premultiplying with $Q^{-1}$ that $M_{\lambda}^{E}=Q M_{\lambda}^{\mathcal{C X}} Q$, or equivalently by postmultiplying with $Q^{-1}$ that $M_{\lambda}^{C}=Q M_{\lambda}^{E} Q$.

We now have the following results for the EANS-value and the corresponding associated game (3.1). 
Lemma 4.6. The EANS-value satisfies the associated game consistency with respect to the associated game $\left\langle N, v_{\lambda}^{E}\right\rangle$, that is $M^{E}=M^{E} M_{\lambda}^{E}$.

Proof. By Proposition 4.1 and Proposition 4.5, we have $M^{C}=M^{C} M_{\lambda}^{C}$ and $M^{E}=Q M_{\lambda}^{C} Q$. Together with (4.9), it follows that

$$
M^{E} M_{\lambda}^{E}=M^{E} Q M_{\lambda}^{C} Q=M^{C} M_{\lambda}^{C} Q=M^{C} Q=M^{E} .
$$

Proposition 4.7. For any game $\langle N, v\rangle \in \mathcal{G}^{N}$, the sequence of repeated associated games $\left\{\left\langle N, v^{k, E}\right\rangle\right\}_{k=1}^{\infty}$ converges, and its limit game is inessential.

Proof. By Proposition 4.5, $M_{\lambda}^{E}=Q M_{\lambda}^{C} Q$ and from the proof of Theorem 4.4 we have that $\lim _{k \rightarrow \infty}\left(M_{\lambda}^{C}\right)^{k}=P D P^{-1}$. Therefore,

$$
\lim _{k \rightarrow \infty}\left(M_{\lambda}^{E}\right)^{k}=Q \lim _{k \rightarrow \infty}\left(M_{\lambda}^{C}\right)^{k} Q=Q P D P^{-1} Q .
$$

So for any game $\langle N, v\rangle$ we have

$$
\lim _{k \rightarrow \infty} v^{k, E}=\lim _{k \rightarrow \infty}\left(M_{\lambda}^{E}\right)^{k} \cdot v=Q P D P^{-1} Q \cdot v .
$$

Since the row-coalitional matrix $P D$ is inessential as shown in the proof of Theorem 4.4, by 2 of Lemma 4.3 we have that $P D P^{-1} Q$ is inessential too. By 3 of Lemma 4.3 it follows that the game $\left\langle N, P D P^{-1} Q \cdot v\right\rangle$ is inessential. Recalling that the dual game of an inessential game is inessential, it follows that the limit game $\left\langle N, Q P D P^{-1} Q \cdot v\right\rangle$ is also inessential.

Theorem 3.1 can now be proved similar to Theorem 3.3. The proof is omitted here.

Theorem 4.4 and Proposition 4.7 claim that both of the two sequences $\left\{\left\langle N, v^{k, C}\right\rangle\right\}_{k=1}^{\infty}$ and $\left\{\left\langle N, v^{k, E}\right\rangle\right\}_{k=1}^{\infty}$ converge to an inessential game, but no exact forms of the limit games are given. However, by the characterization of the EANS and CIS-value respectively, it follows that the sequence $\left\{\left\langle N, v^{k, E}\right\rangle\right\}_{k=1}^{\infty}$ converges to the limit game $\left\langle N, v^{*}\right\rangle$ given by

$$
v^{*}(S)=\sum_{i \in S} v^{*}(\{i\})=\sum_{i \in S} E A N S_{i}(N, v), \text { for all } S \in \Omega .
$$

and the sequence $\left\{\left\langle N, v^{k, C}\right\rangle\right\}_{k=1}^{\infty}$ to the limit game $\left\langle N, v^{* *}\right\rangle$ given by

$$
v^{* *}(S)=\sum_{i \in S} v^{* *}(\{i\})=\sum_{i \in S} C I S_{i}(N, v), \text { for all } S \in \Omega .
$$




\section{Acknowledgements}

This research has been supported by the National Natural Science Foundation of China (Grant Nos. 70901063, 71171163 and 71271171), the Natural Science Basic Research Plan in Shaanxi Province of China (Grant No. 2010JQ1015), and the NPU Foundation for Fundamental Research (Grant No. JC20110276).

\section{References}

Dragan, I., 1991. The potential basis and the weighted Shapley value, Libertas Mathematica 11, 139-150.

Dragan, I., 1996. New mathematical properties of the Banzhaf value, European Journal of Operations Research 95, 451-463.

Driessen, T.S.H., 1991. A survey of consistency properties in cooperative game theory, SIAM Review 33, 43-59.

Driessen, T.S.H., Funaki, Y., 1991. Concidence of and collinearity between game theoretic solutions, OR Spektrum 13, 15-30.

Driessen, T.S.H., 2010. Associated consistency and values for TU games, International Journal of Game Theory 39, 467-482.

Grabisch, M., 2000. The interaction and Moebius representations of fuzzy measures on finite space, $k$-additive measures: a survey, in: Fuzzy Measures and Integrals - Theory and Applications, (Grabisch, M., Murofushi, T., Sugeno M., Kacprzyk J., eds.), pp 70-93.

Hamiache, G., 2001. Associated consistency and Shapley value, International Journal of Game Theory 30, 279-289.

Hamiache, G., 2010. A matrix approach to the associated consistency with an application to the Shapley value, International Game Theory Review $12,175-187$.

Hamiache, G., 2012. A matrix approach to TU games with coalition and communication structures, Social Choice and Welfare 38, 85-100. 
Hernandez-Lamoneda, L., Juarez R., Sanchez-Sanchez, F., 2007. Dissection of solutions in cooperative game theory using representation techniques, International Journal of Game Theory 35, 395-426.

Hwang, Y., 2006. Associated consistency and equal allocation of nonseparable costs, Economic Theory 28, 709-719.

Kleinberg, N.L., Weiss, J.H., 1985. Equivalent $n$-person games and the null space of the Shapley value, Mathematics of Operations Research 10, 233243.

Moulin, H., 1985. The separability axiom and equal sharing method, Journal of Economic Theory 36, 120-148.

Naumova, N.I., 2009. Associated consistency based on utility functions of coalitions, UBS 26, St. Petersburg State University, 79-99.

Thomson, W., 1996. Consistent allocation rules. RCER Working Paper 418, University of Rochester.

van den Brink, R., Funaki, Y., 2009. Axiomatizations of a class of equal surplus sharing solutions for TU-games, Theory and Decision 67, 303-340.

Xu, G., Driessen T., Sun, H., 2008. Matrix analysis for associated consistency in cooperative game theory, Linear Algebra and its Applications 428, 15711586 .

Xu, G., Driessen T., Sun, H., 2009. Matrix approach to dual similar associated consistency for the Shapley value, Linear Algebra and its Applications 430, 2896-2897. 